\title{
O PERFIL DO IDOSO ASSISTIDO EM SERVIÇO \\ UNIVERSITÁRIO AMBULATORIAL
}

\section{The profile of elderly people assisted in an ambulatory university service \\ El perfil del anciano asistido en el servicio universitario dispensarial}

\author{
Jacira Guiro Marino ${ }^{1}$ \\ Márcia Carrera Campos Leal ${ }^{1}$ \\ Ana Paula de Oliveira Marques ${ }^{1}$ \\ Enivaldo Carvalho da Rocha ${ }^{2}$ \\ Paula Tiyemi Shinozaki Mendes ${ }^{3}$
}

\begin{abstract}
RESUMO
Objetivos: Elaborar banco de dados dos idosos assistidos no Núcleo de Atenção ao Idoso - NAI/UFPE; identificar o perfil do idoso em função das variáveis sociodemográficas e de saúde; avaliar o escore obtido no Mini-Exame do Estado Mental (MEEM). Metodologia: Pesquisa quantitativa de natureza exploratóriodescritiva que consistiu na análise de 1.194 prontuários médicos de idosos participantes entre 2000 a 2004. Resultados: Com respeito à distribuição por gênero, $23,4 \%$ dos idosos são do sexo masculino e $76,6 \%$ do feminino. Em relação à escolaridade $60 \%$ tinham menos de quatro anos de estudo. O número de viúvas (38\%) foi três vezes maior que o de viúvos (12\%). Os registros clínicos mais prevalentes sobre a saúde dos idosos foram: problema de visão, hipertensão arterial, antecedente cirúrgico, dislipidemia, tontura, catarata, fratura óssea, reação alérgica e sedentarismo. O desempenho no MEEM apresentou média 24 (DP = 3,7) e mediana 23,5. Destaca-se que 4,9\% dos idosos têm escores menores ou iguais a 10 pontos. Conclusões: Considerando a importância da integralidade na atenção em saúde, os dados investigados poderão subsidiar a estruturação de ações dirigidas à clientela idosa atendida no serviço ambulatorial estudado.

Palavras-chave: idoso; perfil sociodemográfico; MEEM; Ambulatório geronto-geriátrico.
\end{abstract}

\begin{abstract}
Objectives: Develop a database of elder people assisted in the Núcleo de Atenção ao Idoso - NAI/UFPE; identify the profile of the elderly in terms of socio-demographic and health variables; evaluate the score obtained on the Mini Mental State Examination (MMSE). Methodology: Quantitative research of exploratory descriptive nature consisting on the analysis of 1,194 medical records of elderly participants between 2000 and 2004. Results: Concerning the distribution by gender, $23.4 \%$ of the elderly are male and $76.6 \%$ are female. In relation to education, $60 \%$ had less than four years of study. The number of female widows $(38 \%)$ was three times higher than the number of male widows (12\%). The most prevalent clinical records of the elderly people's health were: visual problems, hypertension, surgical history, dyslipidemia, dizziness, cataract, bone fractures, allergic reaction and sedentism. The performance on the MMSE had an average of $24(\mathrm{SD}=3.7)$ and median of 23.5. It was found that $4.9 \%$ of seniors have scored less than 10 points. Conclusions: Considering the importance of completeness in health care, the data investigated can support the structure of actions directed to the elderly patients at the ambulatory place studied.
\end{abstract}

Keywords: elderly; sociodemographic profile; MMSE; geront-geriatric clinic.

1 Departamento de Medicina Social. Universidade Federal de Pernambuco (UFPE). Recife, PE, Brasil. Endereço: Rua Prof. Júlio Ferreira de Melo, 878/1301 - Boa Viagem. 51020-231 - Recife/PE Fone/Fax: (81) 3325-3523. e-mail: jacira@ufpe.br.

2 Departamento de Ciências Sociais. Universidade Federal de Pernambuco. Recife, PE, Brasil.

${ }^{3}$ Departamento de Estatística. Universidade Federal de Pernambuco. Recife, PE, Brasil. 


\section{RESUMEN}

Objetivos: Elaborar un banco de datos de los ancianos asistidos en el Núcleo de Atenção ao Idoso - NAI/ UFPE; identificar el perfil del anciano en función de variables sociales demográficas y de la salud; evaluar el resultado obtenido en un Micro Examen del Estado Mental (MEEM). Metodología: pesquisa de naturaleza de exploración descriptiva que consistió en el análisis de 1194 relatos escritos de los ancianos participantes entre los años de 2000 y 2004. Resultados: Con relación a la distribución por género, 23,4\% de los ancianos son del sexo masculino y $76,6 \%$ del femenino. En relación a la escolaridad $60 \%$ tenían menos de cuatro años de estudio. El número de viudas (38\%) fue tres veces mayor que el de los viudos (12\%). Los registros clínicos más prevalentes sobre la salud de los ancianos fueron: problema de visión, hipertensión arterial, antecedente quirúrgico, dislipidemía, vértigo, catarata, fractura del hueso, reacción alérgica y sedentarismo. El desarrollo en el Micro Examen del Estado Mental presentó una media 24 (DP = 3,7) y mediana 23,5. Destacase que $4,9 \%$ de los ancianos tienen resultados menores o iguales a 10 puntos. Conclusiones: Considerando la importancia integral en la atención en la salud, los datos investigados podrán subsidiar la estructuración de acciones dirigidas a clientela anciana atendida en el servicio del dispensario estudiado.

Palabras-clave: anciano; perfil social demográfico; MEEM; dispensario geronto-geriátrico.

Introdução

Os idosos compõem hoje o segmento populacional que mais cresce em termos proporcionais. De acordo com as projeções estatísticas, até o ano de 2025 seremos a sexta maior população idosa do mundo em números absolutos, com mais de 32 milhões de indivíduos nessa faixa etária, representando cerca de $15 \%$ da população total.

A passagem de uma situação de alta fecundidade e alta mortalidade, para uma de baixa fecundidade, e cada vez mais baixa mortalidade, traduz-se num aumento progressivo da população idosa. Essa transformação demográfica faz-se acompanhar de uma transição epidemiológica, gerando demandas específicas de cuidados dirigidos à população idosa, que responde às alterações do progresso de envelhecimento de maneira diversificada, levando-se em consideração a classe social e o gênero, entre outros.

O aumento expressivo da população idosa demanda uma crescente capacitação de profissionais para a atenção à saúde e ao cuidado específico dessa categoria populacional, considerando as peculiaridades que são intrínsecas ao ser idoso, que apresenta características de uma classe etária que requer cuidados diferenciados, necessitando uma abordagem das múltiplas dimensões.

O miniexame do estado mental (MEEM) se constitui em importante instrumento de rastreio de comprometimento cognitivo, utilizado na avaliação multidimensional do idoso. Como instrumento clínico, pode ser utilizado na detecção de perdas cognitivas, no seguimento evolutivo de doenças e no monitoramento de resposta ao tratamento ministrado. Como instrumento de pesquisa, tem sido largamente empregado em estudos epidemiológicos populacionais.

Visando à programação de ações em saúde e a oferta de serviços adequados à clientela idosa, se faz necessário conhecer o perfil do idoso que o procura. Para isto, foi aprovado em 2004 o Projeto de Extensão Uma análise longitudinal do perfil do idoso atendido pelo Núcleo de Atenção ao Idoso - NAI/UFPE, cujo objetivo era apoiar o importante e valioso trabalho realizado pelo NAI junto à sociedade e reconhecê-lo como exemplo de contribuição social a ser seguido, incentivando a participação de alunos de graduação e de pósgraduação em suas ações de forma a incluir em sua formação uma postura de inserção social e cidadã, integrada às atividades de ensino, pesquisa e extensão. 
Objetivos

Criar um banco de dados dos idosos assistidos no Núcleo de Atenção ao Idoso - NAI/ UFPE.

Identificar perfil do idoso em função das variáveis sociodemográfiicas e de saúde e avaliar o escore obtido no Mini-Exame do Estado Mental (MEEM)

\section{Metodologia}

Baseada na premissa de promover ações para a melhoria da qualidade de vida do idoso, a UFPE implantou o Núcleo de Atenção ao Idoso - NAI, atualmente vinculado a Pró-Reitoria de Extensão da UFPE, presta atendimento em diversas áreas: medicina, odontologia, psicologia, fonoaudiologia, terapia ocupacional, enfermagem, nutrição.

A primeira ação desenvolvida se constituiu de uma plataforma em Acess para resgatar as informações registradas $e$ armazenadas manualmente nos prontuários dos pacientes já atendidos até a data da implantação do projeto, digitando-as em um banco de dados. Dessa forma, passou a ser possível ao NAI acompanhar de forma sistemática as informações sobre os idosos atendidos pelo programa de 2000 a 2004.

A identificação do perfil sociodemográfico e de saúde do idoso foi realizada mediante cruzamento das diversas variáveis para identificar as que estão associadas ao tempo de permanência no NAI.

Neste trabalho é apresentada uma análise sobre as variáveis observadas tais como, sexo, idade, nível de escolaridade, com quem convive, existência de plano de saúde, tempo de ingresso no programa, escore obtido no Mini Exame do Estado Mental - MEEM, dentre outras, além de informações médicas sobre o participante do programa.

Para avaliar a perda cognitiva foi utilizado o Mini-Exame do Estado Mental (MEEM). O MEEM foi escolhido por ser um instrumento psicométrico mundialmente usado para avaliar o estado cognitivo de pessoas adultas e idosas. Utilizou-se a tradução proposta por Bertollucci et al. (1994) e modificada por Almeida OP (1998).

As variáveis sociodemográficas: idade (em anos completos, agrupados por faixa etária), sexo, escolaridade (distribuída em sete categorias: analfabetos, de 1 a 4 anos de estudo, de 5 a 8 anos de estudo e 9 ou mais anos de estudo), estado civil (solteiro/a, casado/a ou com companheiro/a, separado/a e viúvo/a), atividade profissional (categorizada em função da escolaridade requerida para exercê-la), existência de plano de saúde e convivência (tem ou não companhia).

Os registros relativos à saúde contêm informações sobre hipertensão, tonturas, diabetes, dentre várias outras.

Em virtude da existência de dados perdidos (missing values) para algumas variáveis, houve pequena variação no número de informações processadas em determinadas categorias.

\section{Resultados}

No estudo, ora apresentado à proporção de idosos do sexo feminino 76,3\% e a do sexo masculino $23,7 \%$, foi ressaltando a maior procura por serviços de saúde por parte das mulheres. (Tabela 1).

A variação de idade no grupo estudado foi de 60 a 101 anos, sendo a média, 72,7 anos com desvio padrão de 7,95 anos. A idade média dos idosas foi 72,5 anos e dos idosos 74,7 anos. O agrupamento etário nos intervalos de 60 a 
69 anos, 70 a 79 anos, 80 a 89 anos e 90 anos e mais, correspondeu respectivamente a $39,9 \%$ (476), 40,8\% (487), 14,6\% (173) e 3,8\% (45).

Em relação ao estado civil, verificase que $44,8 \%$ dos idosos são casados ou têm companheira, 6,4\% são separados, $16,3 \%$ são solteiros e $32,5 \%$ são viúvos. No entanto, a análise por sexo mostra que dentre os homens, $76,9 \%$ são casados ou têm companheira, enquanto entre as mulheres apenas $35,0 \%$ se enquadram nesta categoria. Situação inversa é observada na condição de viuvez, com um número de mulheres viúvas $(38,9 \%)$ três vezes superior ao número de viúvos (11,9\%), o que confirma a cultura prevalente em nossa sociedade quanto ao recasamento de idosos com mulheres mais jovens e da maior dificuldade de recasamento das mulheres após a viuvez. Destaca-se que 94,4\% (67) dos idosos convivem com alguma companhia, não sendo observada grande diferença por sexo.

Em relação à escolaridade, $60 \%$ dos idosos assistidos no NAI, tinham menos de quatro anos de estudo. Verifica-se, também, que 12,9\% da amostra é constituída por pessoas analfabetas, sendo que 1 a 4 anos de estudo é a situação mais prevalente, correspondendo a $60,8 \%$. A análise por sexo mostra pequena diferença entre homens e mulheres nas faixas de analfabetismo e de nível superior (10,1\% para os homens e $13,7 \%$ para as mulheres e $10,9 \%$ para os homens e $9,0 \%$ para as mulheres, respectivamente).

A distribuição dos idosos em relação à atividade profissional exercida, segundo a escolaridade requerida, foi informada em 642 prontuários, sendo que em $53,9 \%$ deles a condição de alfabetização não se fazia necessária, enquanto que em apenas $4,2 \%$ dos prontuários o nível superior completo era requerido para o desempenho da profissão. Destaca-se que $15 \%$ das mulheres se declararam "do lar", ou seja, não desempenharam atividade profissional ao longo da vida.

Em relação aos dados de saúde, os registros clínicos mais prevalentes sobre os idosos, identificados a partir dos prontuários médicos, considerando ambos os sexos são: hipertensão arterial $(43,0 \%)$, problema de visão $(30,0 \%)$, dislipidemia $(25,0 \%)$, reação alérgica $(17,0 \%)$, antecedente cirúrgico (16,7\%), tontura $(16,5 \%)$, catarata $(14,9 \%)$ e fratura óssea $(14,5 \%)$.

Destaca-se que $18,2 \%$ dos idosos são sedentários, $10 \%$ são fumantes e $6,4 \%$ tem antecedentes de alcoolismo. A frequência de

TABELA 1 - DADOS SÓCIODEMOGRÁFICOS DOS IDOSOS ATENDIDOS NO NAI/UFPE, RECIFE-PE ENTRE 2000 A 2004

\begin{tabular}{|ll|c|c|}
\hline \multicolumn{1}{|c|}{ Variável } & Frequência & $\%$ \\
\hline Sexo & Masculino & 279 & 23,7 \\
& Feminino & 915 & 76,3 \\
& Total & 1194 & \\
\hline Faixa Etária & $60-69$ anos & 476 & 40,3 \\
& $70-79$ anos & 487 & 41,2 \\
& $80-89$ anos & 173 & 14,6 \\
& 90 ou mais & 45 & 3,8 \\
& Total & 1194 & \\
\hline Estado Civil & Casado ou com parceiro & 729 & 45,7 \\
& Separado ou divorciado & 192 & 7,1 \\
& Solteiro & 384 & 15,2 \\
& Viúvo & 1194 & 32,0 \\
& Total & 67 & 5,8 \\
Convivência & Sozinho & 1127 & 94,4 \\
& Acompanhado & 1194 & \\
\hline
\end{tabular}


alcoolismo é 5 vezes maior entre os homens do que entre as mulheres, enquanto que para o tabagismo esta relação é 2,5 vezes.

O desempenho no MEEM, considerando 327 idosos de ambos os sexos para os quais o mesmo foi aplicado, apresentou média de 23,73 (DP = 3,7), mediana 25,0 e moda igual a 30 pontos. Observa-se que $16,2 \%$ (53) idosos têm escore abaixo de 18 pontos, $27,5 \%$ (90) têm entre 18 e 24 e 56,3 (184) acima de 24 pontos. Destaca-se que 4,9\% (18) possuem escores menores ou iguais a 10 pontos. Entre os homens, a média foi 24,35 (DP =6,3) e entre as mulheres 23,41 ( $\mathrm{DP}=5,7)$.

\section{Discussão}

Observou-se, nesse estudo, que a proporção de idosos do sexo masculino $e$ feminino diferiu ligeiramente da proporção esperada na população de idosos na Cidade do Recife. Isto confirma a maior procura das mulheres por assistência à saúde, de forma mais sistemática e contínua ao longo da vida, observada por outros autores e sugerida como um dos fatores explicativos da maior longevidade feminina e responsável pela predominância das mulheres nos serviços de saúde (CHAIMOWICZ, 1998; PEREIRA; CURIONE; VERAS, 2002; SILVESTRE, 1996).

Verificou-se que a freqüência de idosos é mais elevada na faixa etária entre 60 a 69 anos (39,9\%), considerados jovens, de acordo com a classificação adotada por Veras (1994), também é verificado em outros estudos com casuísticas provenientes de serviços de saúde ou de base populacional (PEREIRA; CURIONE; VERAS, 2003; TAVARES; ANJOS, 2003).

Registra-se que a idade média dos idosos é cerca de 2 anos superior a das idosas, e que mais de $80 \%$ dos idosos têm entre 60 e 79 anos.
A análise por sexo mostra uma queda de exigência de escolaridade das mulheres em relação aos homens, em parte justificada, uma vez que $20 \%$ das idosas se enquadram na categoria "do lar".

O número maior de mulheres viúvas em relação aos homens, aspecto também observado em outros estudos, respalda-se em função da maior dificuldade de recasamento após a viuvez, ao contrário do que ocorre em relação aos idosos. Normas sociais e culturais, prevalecentes em nossa sociedade, favorecem aos homens idosos o recasamento com mulheres mais jovens. Este fato, aliado à maior sobrevida feminina, contribui para a existência de um número significativo de mulheres sozinhas, principalmente nas faixas etárias mais avançadas (SILVESTRE et al., 1996; CHAIMOWICZ, 1998; CARVALHO; GARCIA, 2003).

Verificou-se que os idosos que participam do programa apresentam-se com estado mental saudável com exceção de alguns casos. Os dados coletados evidenciam que o processo de envelhecimento não deve ser associado necessariamente à existência de doenças e a degeneração mental.

\section{Conclusão}

Considerando a importância da integralidade na atenção em saúde, os dados investigados poderão subsidiar a estruturação de ações dirigidas à clientela idosa atendida no serviço ambulatorial estudado.

Além de confirmar o impacto do NAI, enquanto unidade de atendimento gerontogeriátrico, a identificação do perfil da clientela idosa demandada poderá contribuir de forma favorável no planejamento e implementação de atividades.

O aumento dos idosos na população implica, em termos de utilização dos serviços 
de saúde, em um maior número de problemas de longa duração, que frequentemente exigem intervenções custosas, envolvendo tecnologia complexa para um cuidado adequado. Em menos de 40 anos, o Brasil passou de um perfil de mortalidade típico de uma população jovem, para um quadro caracterizado por enfermidades complexas e onerosas, próprias das faixas etárias mais avançadas. Esse fato acarreta crescimento das despesas com tratamentos médicos e hospitalares, ao mesmo tempo em que apresenta um desafio para as autoridades sanitárias, especialmente no que tange à implantação de

\section{REFERÊNCIAS}

ALMEIDA OP. Miniexame do estado mental e o diagnóstico de demência no Brasil. Arq Neuropsiquiatr, v. 56, n. 3B, p. 605-612, 1998.

BERTOLUCCI, P. H. F. et al. O Miniexame do Estado Mental em uma população geral: impacto da escolaridade. Arquivos Neuropsiquiátricos, n. 52, p. 1-7, 1994.

CARVALHO J. A. M, GARCIA R. A. O envelhecimento da população brasileira: um enfoque demográfico. Cadernos de Saúde Pública, v. 3, n.19, p. 725-733, 2003.

CHAIMOWICZ, F. Os idosos brasileiros no século XXI: demografia, saúde e sociedade. Belo Horizonte: Postgraduate, $1998.92 \mathrm{p}$.

COELHO FILHO, J. M.; RAMOS, L. Epidemiologia do envelhecimento no Nordeste do Brasil: resultados de inquérito domiciliar. Revista de Saúde Pública, v. 33, n. 5, p. 445-453, out. 1999. novos modelos e métodos de planejamento, gerência e prestação de cuidados (PEREIRA, R. S.; CURIONI C. C; VERAS R., 2003).

Os programas de atenção à saúde do idoso podem ser vistos como aberturas interessantes, com grau de alcance variado, ao investimento em saúde e bem-estar do idoso para além da lógica usual de assistência à doença. Seus méritos e limites devem ser apreciados no processo de desenvolvimento da promoção da saúde do idoso e das estratégias de avaliação de programas nessa área no contexto brasileiro.

PEREIRA, R. S.; CURIONI C. C; VERAS R. Perfil demográfico da população idosa no Brasil e no Rio de Janeiro em 2002. Textos sobre Envelhecimento, v. 1, n. 6, p. 43-59, 2003.

SILVESTRE, J. A.; KALACHE, A.; RAMOS, L. R.; VERAS, R. P. O envelhecimento populacional brasileiro e o setor de saúde. A.G.G., v. 0, n. 1, p. 81-89, set. 1996.

SOARES, J. F; COLOSIMO, E. A. Métodos quantitativos na pesquisa clínica. 40ª RBRAS, Ribeirão Preto, 1995. $105 \mathrm{p}$.

TAVARES E. L; ANJOS, L. A. Perfil antropométrico da população brasileira. Resultados da Pesquisa Nacional sobre Saúde e Nutrição. Cadernos de Saúde Pública, v. 15, n. 4, 1999. Disponível em: <http://www.scielo.br> Acesso em: 21/11/2008.

VERAS, R. P. País jovem com cabelos brancos - a saúde do idoso no Brasil. Rio de Janeiro: Relume Dumará, 1994.

Texto recebido em 16 de fevereiro de 2009. Texto aprovado em 17 de abril de 2009. 